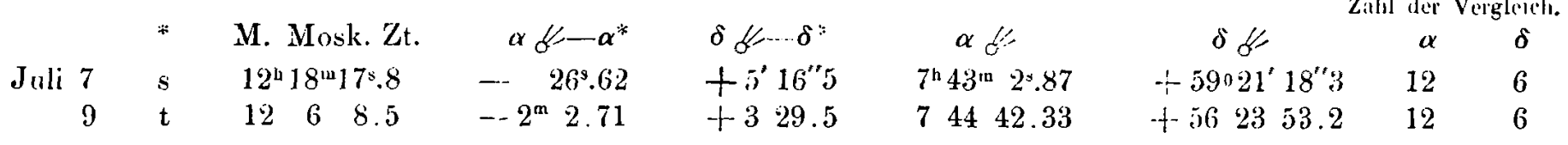

Mittlere Oerter der Vergleichsterne für 1874.0,

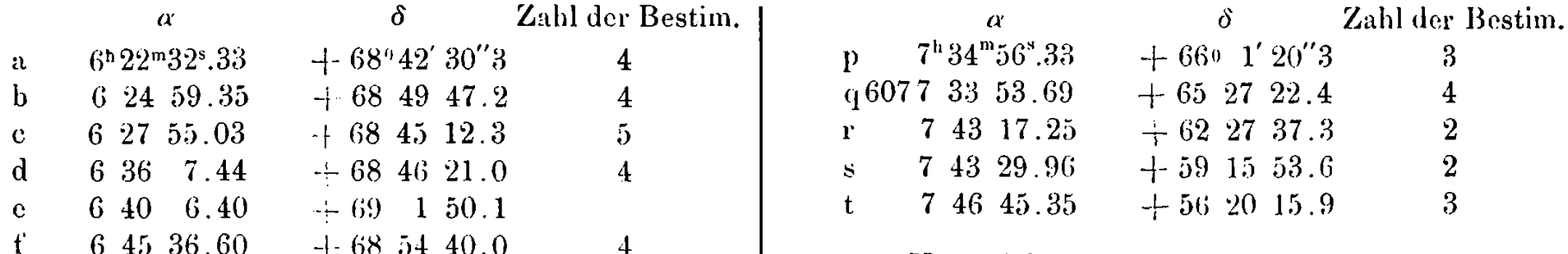

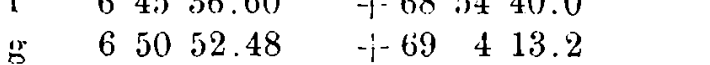

h $\quad 7 \quad 435.86 \quad+685725.2 \quad 4$

i $\quad 7 \quad 7037.60 \quad+k$ (is $85148.4 \quad 4$

j $7.1453 .41 \quad+684622.4 \quad$ j

k $\quad$ T2042.26 $\quad-6822 \quad 4.2 \quad 5$

lscq. $72256.71 \quad-1.6743 \quad 12.5 \quad 5$

m $72933.97+673213.8$ 4

n $\quad 7 \quad 2839.69 \quad-1672054.5 \quad 4$

$0 \quad 73147.50 \quad-1 \cdots 664436.5 \quad 2$

Die Vergleichsterne, - d und e ausgenommen, sind am Meridiankraise der hiesigen Sternwarte von I. Gromerratii sehr genau bestimmt. Den Stern d habe ich micrometrisch bostimmt: der Stern e $=43$ Cammelopardi. Die Beobachtungen. des Cometen sind ron mir an grossen Refractor: bei 120 facher Vergrösserung, angestellt.

Moskau, den 12. October 1874.

Prof. T. Brediclein.

Parallelsterne ans der "Zone 6.5" $70^{\prime \prime}$ des von Coggia 1874 April 17 entdeckten Cometen.

\begin{tabular}{|c|c|c|}
\hline Fo. & Gri:sse. & AR. 1855.0 \\
\hline 1 & 9.0 & $6^{\mathrm{h}} 19^{\mathrm{m}} 30^{\mathrm{m}} .44$ \\
\hline 2 & 7.8 & 2238.89 \\
\hline 3 & 8.0 & 2312.80 \\
\hline 4 & 8.9 & $25 \quad 6.06$ \\
\hline$\vdots$ & 8.0 & $28 \quad 1.66$ \\
\hline 6 & 8.0 & 3240.35 \\
\hline 7 & 8.5 & $36 \quad 17.77$ \\
\hline 8 & 8.0 & 3750.56 \\
\hline 9 & 7.7 & 4125.85 \\
\hline 10 & 7.5 & 4343.07 \\
\hline 11 & 7.6 & $44 \quad 49.69$ \\
\hline 12 & 7.4 & $45 \quad 43.11$ \\
\hline 13 & 8.8 & 4636.53 \\
\hline 14 & 8.9 & $47 \quad 5.25$ \\
\hline 15 & 8.4 & $48 \quad 0.09$ \\
\hline 16 & 7.2 & $49 \quad 8.02$ \\
\hline 17 & 8.0 & 5059.08 \\
\hline * 18 & 8.4 & 5656.13 \\
\hline 19 & 8.3 & 5715.98 \\
\hline 20 & 8.9 & 65954.67 \\
\hline 21 & 7.0 & $\begin{array}{lll}7 & 133.67\end{array}$ \\
\hline 22 & 8.8 & 442.37 \\
\hline 23 & 9.5 & 459.67 \\
\hline
\end{tabular}

$\begin{array}{crc}\text { Decl. } & 187.5 .0 \\ 69024 & 12.0 \\ 68 & 42 & 29.3 \\ 69 & 26 & 59.5 \\ 68 & 49 & 45.9 \\ 68 & 45 & 11.1 \\ 69 & 45 & 8.9 \\ 69 & 37 & 22.8 \\ 68 & 21 & 12.0 \\ 68 & 23 & 48.4 \\ 68 & 20 & 44.9 \\ 68 & 31 & 12.8 \\ 68 & 54 & 36.7 \\ 68 & 31 & 14.1 \\ 68 & 43 & 19.4 \\ 68 & 37 & 50.1 \\ 69 & 22 & 36.5 \\ 69 & 4 & 10.4 \\ 68 & 46 & 39.3 \\ 69 & 5 & 14.6 \\ 69 & 11 & 19.9 \\ 69 & 0 & 31.0 \\ 68 & 57 & 21.7 \\ 68 & 57 & 21.8\end{array}$

Dect. 1875.0

$69024^{\prime} 12^{\prime \prime} 0$

$68 \quad 4945.9$

$\begin{array}{llll}69 & 37 & 22.8\end{array}$

682348.

$\begin{array}{llll}68 & 20 & 44.9\end{array}$

$\begin{array}{llll}68 & 31 & 12.8\end{array}$

$68 \quad 31 \quad 14.1$

$\begin{array}{llll}68 & 43 & 19.4\end{array}$

$692 \times 36.5$

$\begin{array}{llll}69 & 4 & 10.4\end{array}$

$\begin{array}{llll}68 & 46 & 39.8\end{array}$

$69 \quad 5 \quad 14.6$

$\begin{array}{lll}69 & 11 & 19.9\end{array}$

$\begin{array}{llll}68 & 57 & 21.7\end{array}$

$\begin{array}{llll}68 & 57 & 21.8\end{array}$

\begin{tabular}{|c|c|c|}
\hline$N_{0}$. & Grösse. & AR. 1875.0 \\
\hline 24 & 7.0 & $7^{1+} \quad 7^{m} 21^{9} .25$ \\
\hline 25 & 8.9 & 828.16 \\
\hline 20 & 8.9 & 1044.13 \\
\hline 27 & 7.9 & $11 \quad 8.32$ \\
\hline 28 & 9.0 & $12 \quad 6.09$ \\
\hline$* 29$ & 9.2 & 1459.84 \\
\hline 30 & 8.4 & 159.80 \\
\hline$* 31$ & 8.8 & $2035.3 i i$ \\
\hline 32 & 9.0 & $22 \quad 30.44$ \\
\hline 33 & 8.6 & $23 \quad 2.75$ \\
\hline 34 & 7.7 & $24 \quad 27.4 j$ \\
\hline$\because 35$ & 8.7 & 2625.81 \\
\hline$: 36$ & 7.8 & $26 \quad 33.87$ \\
\hline$: 37$ & 8.7 & 2845.63 \\
\hline 38 & 8.0 & $29 \begin{array}{lll}29 & 12 & 39\end{array}$ \\
\hline 39 & 8.1 & $29 \quad 33.86$ \\
\hline 40 & -8.8 & $30 \quad 39.11$ \\
\hline 41 & 8.2 & 3055.56 \\
\hline 42 & 8.1 & 3059.11 \\
\hline$\because 43$ & 9.3 & 3132.95 \\
\hline 44 & 9.0 & 3146.82 \\
\hline 45 & 8.5 & 3153.66 \\
\hline 46 & 8.4 & $32 \quad 6.84$ \\
\hline
\end{tabular}

Dect. 1875.0

$\left.68^{\prime \prime} 4\right)^{\prime} 45^{\prime \prime} 1$

$68 \quad 42 \quad 31.0$

$\begin{array}{llll}68 & 51 & 41.9\end{array}$

$\begin{array}{llll}68 & 8 & 43.5\end{array}$

$6859 \quad 11.6$

$\begin{array}{llll}68 & 46 & 10,4\end{array}$

$67 \quad 5454.7$

(is 3948.8

$\begin{array}{lll}67 & 41 & 5 \% .4\end{array}$

$\begin{array}{lll}67 & 43 & 5.8\end{array}$

6056 35.0

6(j) $50 \quad 34.9$

$\begin{array}{lll}67 & 19 & 33.0\end{array}$

$\begin{array}{llll}67 & 20 & 48.4\end{array}$

$\begin{array}{llll}67 & 50 & 59.5\end{array}$

$\begin{array}{lll}66 & 30 & 39.0\end{array}$

$67 \quad 53 \quad 42.6$

$\begin{array}{llll}65 & 17 & 13.2\end{array}$

65 $34 \quad 32.8$

$\begin{array}{lll}66 & 29 & 28.3\end{array}$

654250.4

$66 \quad 44 \quad 26.5$

65 4339.1 


\begin{tabular}{|c|c|c|c|}
\hline$o$. & Grïssc. & AR. 1875.0 & e. 1875 \\
\hline 47 & 9.1 & $7^{\mathrm{h}} 32^{\mathrm{w}} 32^{\mathrm{s}} .02$ & $65^{n} 27^{\prime} \quad 9^{\prime \prime} 8$ \\
\hline & 9.1 & $33 \quad 2.78$ & $\left(\begin{array}{llll}35 & 21 & 41 .\end{array}\right.$ \\
\hline & 8.0 & 3317.71 & 945 \\
\hline 50 & 8.0 & 3356. & $\begin{array}{lll}65 & 0 & 42\end{array}$ \\
\hline 51\{ & $\begin{array}{l}7.5 \\
7.9\end{array}$ & 3359.34 & $6 \mathbf{5} 27$ \\
\hline & 6.5 & 3442. & 6545 \\
\hline$b$ & 2 & $35 \quad 2$. & $66 \quad 110$ \\
\hline 5 & 9.3 & $35 \quad 54$ & $\begin{array}{lll}67 & 0 & 26.1\end{array}$ \\
\hline 5 & 9 . & $35:$ & 6638 \\
\hline je & 9.0 & 365 & 6745 \\
\hline 5 & 9.0 & 3836.70 & $\begin{array}{lll}66 & 5 & 39.3\end{array}$ \\
\hline 58 & 9.1 & $39 \quad 32.80$ & 6635 \\
\hline
\end{tabular}

Dic obenstehenden, von Observator Gelmuydme reducirten mittleren Uerter einiser in unseren Zonen $\left(65^{\circ}\right.$ bis $\left.70^{\circ}\right)$ in beiden Kreislagen beobachteten Sterne werden hoffentlich fïr die definitive Bearbeitung der Beobachtungen des grossen von Coygia entdeckten Cometen von einigem Nutzen werden. Diesen Positionen liegt die "nene Bestimmung der mittleren Oerter der Zusatzsterne nacil Beobachtumgen inf der lulkowaer Sternwarte" zu Grunde ( 1 . J. S. der Astr. Gesellsch. IX. Band, 2. Heft). Das war aber nicht der Fall mit den fribher I'rm Geheimrath Argelander mitgetheilten, von ihm in Astr. Nachr. No. 2014 citirten Oertern für 1874.0, welche daher hicr fïr 1875.0 wieder erscheinen. - Nur die mit * bezeicineten Sterne siud in B. D. olne Angabe früherer Beobachtung angeführt. - No. 37, 40 and 57 sind noch nicht mit genïgender Sicherheit bestimunt. No. 51 ist duplex (Medium).

Christiania Stcrnwarte, 1874, Nov. 17.

(. Fearnley.

\title{
Elemente und Ephemeride des Planeten (139).
}

Aus den Beobachtungen Pola Oct. 13, Berlin Oct. $28: 12^{\mathrm{h}} \mathrm{m}$. B. 7t.

und Pola Nov. 11 leitete ich die folgenden Elcmente ab, die mit den von Prof. Tietjen gegebenen sehr nahe ïbereinstimmen :

1874 Dec. 1.5 mittl. Berl. Zt.

$$
\begin{aligned}
& M==71^{\prime \prime} 56^{\prime} 15^{\prime \prime} 1 \\
& \omega=\begin{array}{lll}
197 & 18 & 28.3
\end{array} \\
& \delta=1071437.0 \text { mittl. Aer. } 1874.0 \\
& \left.i=\begin{array}{lll}
3 & 94.3
\end{array}\right) \\
& \varphi=112446.3 \\
& \mu=796.693 \\
& \log \|=0.432477
\end{aligned}
$$

Mit diesen Elementen rechnete ich die folgende Ephemeride:

$12 \mathrm{~h}$ in. B. Zt. 1874 Dec. 1

$$
\begin{array}{lr}
" & 2 \\
" & 3 \\
. & 4 \\
" & 5 \\
" & 6 \\
" & 7 \\
" & 8 \\
" & 9 \\
" & 10
\end{array}
$$

$$
\alpha \text { app. }
$$$$
\delta \text { app. }
$$$$
\text { 1" } 33^{m} 51^{s} .5
$$$$
\text { -f } 5^{0} 19^{\prime} 32^{\prime \prime}
$$$$
3340.3
$$$$
3330.6
$$$$
3322.5
$$$$
\begin{array}{lll}
33 & 15.9
\end{array}
$$$$
\begin{array}{lll}
33 & 10.9
\end{array}
$$$$
33 \quad 7.4
$$$$
\begin{array}{ll}
33 & 5.4
\end{array}
$$$$
33 \quad 5.0
$$$$
1336 .
$$

2140

2249

$24 \quad 6$

$25 \quad 32$

$27 \quad 7$

$28 \quad 52$

$30 \quad 45$

-553246

$$
\text { , } 10
$$

0.28141

$\operatorname{Iog} \triangle$

0.27021

0.29287
1874 Dec. 11

, 12

$\because 13$

,. 14

., 15

, 16

, 17

, 18

, 19

, 20

, 21

, . 22

, 23

, 224

., 25

, 26

$\because 27$

, 28

, 29

$\alpha$ app.

$1^{11} 33^{\mathrm{m}} 8^{\mathrm{s}} .6$

$\begin{array}{lll}33 & 12.7\end{array}$

3318.2

$\begin{array}{lll}33 & 25.2\end{array}$

3333.7

3343.6

3355.0

$\begin{array}{ll}34 & 7.7\end{array}$

$34 \quad 21.9$

3437.5

3454.4

3512.6

35 32.2

35 53.0

$36 \quad 15.2$

3638.7

$37 \quad 3.4$

3729.4

3756.6

3825.1

3854.7

3925.5

13957.6

1875 Jan. 1

$\begin{array}{rr}, & 31 \\ , \quad & 1 \\ \text { an. } & 1\end{array}$

Wien, den $\delta$ app.

$+5034^{\prime} 57^{\prime \prime}$

$37 \quad 15$

$39 \quad 42$
4

42. 18

$\begin{array}{ll}45 & 1\end{array}$

$47 \quad 52$

5052

$53 \quad 59$

$+55713$

$+6035$

$4 \quad 4$

740

1123

$15 \quad 12$

19) 9

2312

$\begin{array}{ll}27 & 21\end{array}$

3137

$35 \quad 59$

$40 \quad 26$

$\begin{array}{ll}45 & 0\end{array}$

4940

0.31628

0.30452

0.31628

0.32808

0.33985

0.35155

$+65425$

0.36315

$\operatorname{Jog} \triangle$

0

政

